\title{
Boundary Conditions as Mass Generation Mechanism for Real Scalar Fields
}

\author{
José Alexandre Nogueira and Pedro Leite Barbieri \\ Departamento de Física, Centro de Ciências Exatas, \\ Universidade Federal do Espirito Santo, \\ 29.060-900 - Vitória-ES - Brazil, \\ E-mail:nogueira@cce.ufes.br
}

Received on 10 December, 2001. Revised version received on 7 March, 2002.

\begin{abstract}
We consider the effects of homogeneous Dirichlet's boundary conditions on two infinite parallel plane surfaces separated by some small distance $a$. We find that although spontaneous symmetry breaking does not occur for the theory of a massless, quartically self-interacting real scalar field, the theory becomes a theory of a massive scalar field.
\end{abstract}

\section{Introduction}

The origin of particle mass has been a puzzle for theoretical physicists. In the context of the standard model, the Higgs mechanism is well accepted as the mass generation mechanism. The underlying idea is that the universe is filled with a field, the Higgs field. The spin-zero Higgs field is a doublet in the $\mathrm{SU}(2)$ space and carries non-zero hypercharge, and it is a singlet in the $\mathrm{SU}(3)$ space of color.

Bosonic gauge and fermionic matter fields acquire mass from their interactions with the Higgs field. It is of fundamental importance to this mechanism that excited states (i. e., with one or more Higgs) are not orthogonal to the ground state (i. e., vacuum). Since states with one or more Higgs carry non-zero SU(2) and $\mathrm{U}(1)$ quantum numbers then they are non-zero for the vacuum as well. As a consequence of this the $\mathrm{SU}(2)$ and U(1) symmetries are spontaneously broken. The Lagrangian is symmetric under $\mathrm{SU}(2)$ and $\mathrm{U}(1)$ transformations but the vacuum is not [1]. Technically this is achieved introducing a Higgs potential of imaginary mass and quartic interaction.

In the Coleman-Weinberg alternative approach the spontaneous symmetry breaking is induced by 1-loop radiative corrections and the mass being vanishing in the tree approximation [2]. In the massless scalar electrodynamics, although the vacuum expectation value of the classical potential is unique (classically $\langle\phi\rangle=0$ ), in its renormalized form the effective potential in 1-loop becomes degenerate $(\langle\phi\rangle$ is arbitrary). The mass renormalization condition fixes a determined vacuum and breaks the symmetry of the theory. The spontaneous symmetry breaking leads both scalar and vector particles to dynamically acquire mass whose value is proportional to the vacuum expectation value. This is only possible because there are two parameters in the theory: the electromagnetic coupling constant, $e$, and the coupling constant of the quartic self-interaction, $\lambda$, which must be of order $e^{4}$.

In a theory of massless real scalar field the existence of just one parameter does not allow a non-zero vacuum expectation value and the theory remains massless.

Boundary conditions do not affect the classical potential, but quantum corrections are affected and the effective potential changes. This may cause effects on the quantum vacuum expectation value $\langle\phi\rangle$. Moreover boundary conditions introduce a new parameter, the length of the finite region. With these considerations we investigate the effects of boundary conditions on the vacuum expectation value $\langle\phi\rangle=0$ and the mass generation. Besides the introduction of a new parameter makes more attractive the real scalar field theory.

The study of boundary conditions effects in Quantum Field Theory is not new. The Casimir effect [3], which is the change of the vacuum energy density due to constraints on the quantum field, induced by boundary conditions in space-time was experimentally observed in 1958 [4] and recently [5, 6], and a lot of applications have been accomplished [7 - 10], such as: gravity models, black holes, bag models, nonlinear meson theories describing baryons as solitons, the cosmological constant problem, compactification of the extra dimensions in Kaluza-Klein theories, quantum liquids [11], condensed matter [12].

In this work we study the effects of the boundary conditions on a massless real scalar field with quartic self-interaction which satisfies homogeneous Dirichlet 
conditions on two infinite parallel plane surfaces separated by some small distance $a$. The study of the $\lambda \phi^{4}$ theory is very important in face of its applications in the Weinberg-Salam model of weak interactions, fermions masses generation, in solid state physics [13], inflationary models [14], solitons [15] and Casimir effect [16].

The effects of the boundary conditions on a $\lambda \phi^{4}$ theory were firstly considered, until where we are concerned, by Ford [17], Ford and Yoshimura [18] and Tom [19]. They found that a massless scalar field at the tree level could develop a mass at the one-loop level as a consequence of both the $\lambda \phi^{4}$ self-interaction and the boundary conditions. In this work we show that if the length of the finite region is small enough the theory will became one massive at the order $\hbar^{0}$. That is because $\lambda$ and the length of the region finite, $a$, are independent parameters and the second derivative of the effective potential become of the order $\hbar^{0}$, even though it is a one-loop result, then it can not be subtracted out by the mass counterterm of the order $\hbar$. So there is a physical mass (measure) and we can not set the renormalized mass to zero. Hence boundary conditions are a possible mass generation mechanism when the length of finite region is small enough. We understand $a$ is small enough when there is a typical length scale where $a^{2}$ is of the order $\lambda$. Therefore that typical length scale determines the mass scale of theory.

It is important to stress that we do not use the imaginary mass term of the Higgs potential in order to avoid that it induces spontaneous symmetry breaking. There- fore with the purpose to evaluate the actual effects of boundary conditions we consider the real scalar field, with a second parameter introduced by the boundary conditions.

As will be seen subsequently the boundary conditions do not induce a degenerate vacuum state, although there is a typical length scale of the finite region where the massless scalar field acquires mass.

The outline of the paper is as follows: Section 2 is a brief review of how to calculate the effective potential at 1-loop. In section 3 we calculate the effective potential for massless real scalar field, and evaluate the vacuum state and the renormalized mass. In contrast to [19], we derive the effective potential at the one-loop level as a sum of modified Bessel functions, then we take the length of the finite region, $a$, very small to expand each term of the sum in power of $a$ and finally throw away higher-order terms. In section 4 we point out our conclusions and some speculations.

\section{Effective Potential}

In the functional method approach of quantum field theory, the effective potential is found as a loop expansion (or equivalently in powers of $\hbar$ ), that is, its classical amount plus quantum corrections [20 - 26].

Let $\phi(x)$ be a single real scalar field in a Minkowski space-time, subjected to the potential $V(\phi)$. The effective potential to the first order in the loop expansion is given by

$$
V_{e f}\left(\phi_{c}\right)=V_{c l}\left(\phi_{c}\right)+\frac{1}{2} \frac{\hbar}{\Omega} \ln \operatorname{det}\left[\frac{\delta^{2} S\left[\phi_{c}\right]}{\delta \phi(x) \delta \phi(y)}\right]=V_{c l}\left(\phi_{c}\right)+V_{e f}^{(1)}\left(\phi_{c}\right)
$$

where the classical field $\phi_{c}$ is the vacuum expectation value in the presence of an external source $J(x)$, taken as a constant value $\phi_{c}=\rho$, therefore, in the limit $J \rightarrow 0, S[\phi]$ is the classical action and $\Omega$ is the four dimensional space-time volume.

Performing the analytic continuation to the Euclidean space-time [21 - 23], the classical action can be written as

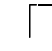

(1)

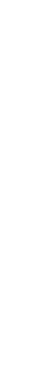

$$
S[\phi]=\int d^{4} x\left[\frac{1}{2} \partial_{\mu} \phi \partial_{\mu} \phi+V_{c l}(\phi)\right],
$$

where the Euclidean summation convention is assumed for repeated indexes. From Eq.(2) we get the matrix $m(x, y)$ of the quadratic variation of the action $S[\phi]$

$$
m(x, y) \equiv \frac{\delta^{2} S[\phi]}{\delta \phi(x) \delta \phi(y)}=\delta^{4}(x-y)\left[-\delta^{\mu \nu} \partial_{\mu} \partial_{\nu}+V_{c l}^{\prime \prime}(\phi)\right]
$$


Due to the Euclidean analytic continuation the operator $m$ is a real, elliptical and self-adjoint. For operators with these properties we define a generalized zeta function [7, 8, 23 and 27]. If $\left\{\lambda_{i}\right\}$ are the eigenvalues of the operator $m(x, y)$, then the generalized zeta function associated to $M(x, y)\left(m \rightarrow M=\frac{m}{\mu^{2}}\right)$ is defined by

$$
\zeta_{M}(s)=\sum_{i}\left(\frac{\lambda_{i}}{\mu^{2}}\right)^{-s}
$$

where we have introduced an unknown scale parameter $\mu$, with dimensions of (length) ${ }^{-1}$ or mass in order to keep the zeta function dimensionless for all s. The introduction of the scale parameter $\mu$, can be best understood when we observe that a hidden splitting of the divergent integral there is in the proceeding of zeta function regularization, that is, a separation of the divergent and finite parts of the $V_{e f}\left(\phi_{c}\right)$ ( on page 208 [23] and on page 88 [24]). It is well-known the relation [21]

$$
\ln \operatorname{det} M=-\frac{d \zeta_{M}(0)}{d s}
$$

Now, the effective potential in the one-loop approximation can be written as

$$
V_{e f}\left(\phi_{c}\right)=V_{c l}\left(\phi_{c}\right)-\frac{1}{2} \frac{\hbar}{\Omega} \frac{d \zeta_{M}(0)}{d s} .
$$

Due to the regularity of the generalized zeta function at $s=0$ [27], the evaluation of the effective potential (Eq.(6)) gives a finite result with no need of substraction of any pole, or addition of infinite counterterms. Evidently, the fitting of the theory parameters taking into account the observed results, leads to the renormalization conditions

$$
\begin{gathered}
\left.\frac{d^{2} V_{e f}}{d \phi_{c}^{2}}\right|_{\phi_{c}=\langle\phi\rangle}=m_{R}^{2}, \\
\left.\frac{d^{4} V_{e f}}{d \phi_{c}^{4}}\right|_{\phi_{c}=\langle\phi\rangle}=\lambda_{R},
\end{gathered}
$$

where $m_{R}$ is the renormalized mass, $\lambda_{R}$ is the renormalized coupling constant and $\langle\phi\rangle$ is the minimum of the effective potential (subtraction or renormalization point) [25]. Since $\phi_{c}$ takes on value $\langle\phi\rangle$ in the ground state, then $\langle\phi\rangle$ is called the vacuum expectation value of $\phi,\langle\phi\rangle=\langle 0|\phi| 0\rangle$.

In the case of theories of null mass, the subtraction point for the renormalization condition (8) cannot be taken at $\langle\phi\rangle=0$ due to the logarithmic singularity. Even so in that case there is no intrinsic mass scale; therefore all the renormalization points are equivalent and the condition (8) is replaced by

$$
\left.\frac{d^{4} V_{e f}}{d \phi_{c}^{4}}\right|_{\phi_{c}=M}=\lambda_{R}
$$

where $M$ is a arbitrary floating renormalization point [23].

\section{Mass Generation}

Now, let us consider the theory of a massless, quartically self-interacting real scalar field $\phi(x)$ satisfying homogeneous Dirichlet's boundary conditions on two infinite parallel plane surfaces separated by some small distance $a$.

The Lagrangian density for this theory is

$$
\mathcal{L}=\frac{1}{2} \partial_{\mu} \phi \partial^{\mu} \phi-\frac{\lambda}{4 !} \phi^{4}
$$

The Lagrangian above is even in $\phi$, so it is invariant under discreet symmetry (G-parity) defined by the transformation $\phi \rightarrow-\phi$.

In this case the zeta function, defined by Eq.(4), is given by $[7,8,24,28-33]$

$$
\zeta_{m}(s)=\sum_{N=1}^{\infty} \int_{-\infty}^{+\infty} \frac{\Omega}{(2 \pi)^{3} a} d^{3} k\left[k^{2}+\frac{\pi^{2} N^{2}}{a^{2}}+\frac{\lambda}{2} \phi_{c}^{2}\right]^{-s} .
$$

Using the integral [28]

$$
\int_{-\infty}^{+\infty}\left[k^{2}+A^{2}\right]^{-s} d^{m} k=\frac{\pi^{\frac{m}{2}} \Gamma\left(s-\frac{m}{2}\right)}{\Gamma(s)}\left(A^{2}\right)^{\frac{m}{2}-s}
$$

and using the formula [34]

$$
\sum_{N=1}^{\infty}\left[N^{2}+B^{2}\right]^{-p}=-\frac{1}{2} B^{-2 p}+\frac{\pi^{\frac{1}{2}}}{2 B^{2 p-1} \Gamma(p)}\left[\Gamma\left(p-\frac{1}{2}\right)+4 \sum_{N=1}^{\infty} \frac{K_{p-\frac{1}{2}}(2 \pi N B)}{(N \pi B)^{\frac{1}{2}-p}}\right]
$$

where $K_{\nu}(x)$ are modified Bessel functions, we get

$$
\zeta_{m}(s)=-\frac{\Omega}{16 \pi^{\frac{3}{2}} a} \frac{\Gamma\left(s-\frac{3}{2}\right)}{\Gamma(s)}\left(\alpha \phi_{c}\right)^{3-2 s}+
$$




$$
\begin{gathered}
+\frac{\Omega}{16 \pi^{2}} \frac{1}{(s-1)(s-2)}\left(\alpha \phi_{c}\right)^{4-2 s}+ \\
+\frac{\Omega}{4 \pi^{2}} \frac{\left(\alpha \phi_{c}\right)^{4-2 s}}{\Gamma(s)} \sum_{N=1}^{\infty} \frac{K_{s-2}\left(2 N \alpha a \phi_{c}\right)}{\left(N \alpha a \phi_{c}\right)^{2-s}}
\end{gathered}
$$

where we define $\alpha^{2}=\frac{\lambda}{2}$ for the sake of simplicity.

In order to calculate the one-loop effective potential, we compute $\zeta_{m}(0)$ and $\zeta_{m}^{\prime}(0)$ from Eq.(14) and use them in Eq. $(6)^{1}$

$$
\begin{gathered}
V_{e f}\left(\phi_{c}\right)=\frac{2 \alpha^{2}}{4 !} \phi_{c}^{4}+\frac{\hbar}{24 \pi a} \alpha^{3} \phi_{c}^{3}+\frac{\hbar}{64 \pi^{2}} \alpha^{4} \phi_{c}^{4}\left[\ln \left(\frac{\alpha^{2} \phi_{c}^{2}}{\mu^{2}}\right)-\frac{3}{2}\right]+ \\
-\frac{\hbar}{8 \pi^{2}} \alpha^{4} \phi_{c}^{4} \sum_{N=1}^{\infty} \frac{K_{2}\left(2 N \alpha a \phi_{c}\right)}{\left(N \alpha a \phi_{c}\right)^{2}}
\end{gathered}
$$

recalling that $K_{\nu}(x)=K_{-\nu}(x)$. We notice that, as we have to take absolute value of $\phi_{c}$ in the formula (13), the second term on the right-hand side of Eq.(15) does not yield symmetry breaking.

Let $m \geq 0$ be an integer number, such that, $2 m \alpha a \phi_{c}<1$ and $2(m+1) \alpha a \phi_{c} \geq 1$. Then, the sum in Eq.(15) can be write as

$$
\begin{gathered}
\frac{\hbar}{8 \pi^{2}} \alpha^{4} \phi_{c}^{4} \sum_{N=1}^{\infty} \frac{K_{2}\left(2 N \alpha a \phi_{c}\right)}{\left(N \alpha a \phi_{c}\right)^{2}}= \\
=\frac{\hbar}{8 \pi^{2}} \alpha^{4} \phi_{c}^{4} \sum_{N=1}^{m} \frac{K_{2}\left(2 N \alpha a \phi_{c}\right)}{\left(N \alpha a \phi_{c}\right)^{2}}+\frac{\hbar}{8 \pi^{2}} \alpha^{4} \phi_{c}^{4} \sum_{N=m+1}^{\infty} \frac{K_{2}\left(2 N \alpha a \phi_{c}\right)}{\left(N \alpha a \phi_{c}\right)^{2}} .
\end{gathered}
$$

Since $2 N \alpha a \phi_{c}<1$, for any $N \leq m$, we expand the first term on the right-hand side of Eq.(16) using the relation $[35-39]$

$$
\begin{gathered}
\left(\frac{x}{2}\right)^{\nu} K_{\nu}(x)=\frac{1}{2} \sum_{a=0}^{\nu-1}(-1)^{a}\left(\frac{x}{2}\right)^{2 a} \frac{\Gamma(\nu-a)}{\Gamma(a+1)}+ \\
+\sum_{a=0}^{\infty} \frac{(-1)^{\nu}\left(\frac{x}{2}\right)^{2 \nu+2 a}}{\Gamma(a+1) \Gamma(\nu+a+1)}\left[\psi(a+1)+\psi(\nu+a+1)-2 \ln \left(\frac{x}{2}\right)\right],
\end{gathered}
$$

for $\nu>0$, to get

$$
\begin{gathered}
\frac{\hbar}{8 \pi^{2}} \alpha^{4} \phi_{c}^{4} \sum_{N=1}^{\infty} \frac{K_{2}\left(2 N \alpha a \phi_{c}\right)}{\left(N \alpha a \phi_{c}\right)^{2}}=\frac{\hbar}{16 \pi^{2} a^{4}} \sum_{N=1}^{m}\left(\frac{1}{N}\right)^{4}-\frac{\hbar}{16 \pi^{2} a^{2}} \alpha^{2} \phi_{c}^{2} \sum_{N=1}^{m}\left(\frac{1}{N}\right)^{2}+ \\
-\frac{\hbar}{16 \pi^{2}} \alpha^{4} \phi_{c}^{4} \sum_{N=1}^{m}\left[\ln \left(N \alpha a \phi_{c}\right)+\gamma-3 / 4\right]+\frac{\hbar}{8 \pi^{2}} \alpha^{4} \phi_{c}^{4} \sum_{N=1}^{m} \mathcal{O}\left(N \alpha a \phi_{c}\right)^{2}+ \\
+\frac{\hbar}{8 \pi^{2}} \alpha^{4} \phi_{c}^{4} \sum_{N=m+1}^{\infty} \frac{K_{2}\left(2 N \alpha a \phi_{c}\right)}{\left(N \alpha a \phi_{c}\right)^{2}}
\end{gathered}
$$

where $\gamma$ is the Euler number.

The first sum on the right-hand side of Eq.(16) exists, provided $\bar{a}(\bar{a}=a \mu)$ be of order $\alpha^{n}(n \geq 0)$, since $\bar{\phi}_{c}$ $\left(\bar{\phi}_{c}=\frac{\phi_{c}}{\mu}\right)$ must be of order $\alpha^{0}$. Hence, up to higher-order terms, the effective potential becomes

$$
V_{e f}\left(\phi_{c}\right)=\frac{2 \alpha^{2}}{4 !} \phi_{c}^{4}+\frac{\hbar}{24 \pi a} \alpha^{3} \phi_{c}^{3}-\frac{\hbar}{16 \pi^{2} a^{4}} \sum_{N=1}^{m}\left(\frac{1}{N}\right)^{4}+\frac{\hbar}{16 \pi^{2} a^{2}} \alpha^{2} \phi_{c}^{2} \sum_{N=1}^{m}\left(\frac{1}{N}\right)^{2} .
$$

For large $m$, we may approximate the two sums in Eq.(19) to Riemann zeta functions and to obtain

$$
V_{e f}\left(\phi_{c}\right)=\frac{2 \alpha^{2}}{4 !} \phi_{c}^{4}+\frac{\hbar}{24 \pi a} \alpha^{3} \phi_{c}^{3}-\frac{\hbar \pi^{2}}{1440 a^{4}}+\frac{\hbar \alpha^{2} \phi_{c}^{2}}{96 a^{2}}
$$

\footnotetext{
${ }^{1}$ We keep $\hbar$ to mark the quantum corrections, but we set $\hbar=c=1$ everywhere else.
} 
The minimum occurs at $\phi_{c}=\langle\phi\rangle$, where

$$
\left.\frac{d V_{e f}}{d \phi_{c}}\right|_{\phi_{c}=\langle\phi\rangle}=0 .
$$

Differentiating Eq.(20), we have

$$
\langle\phi\rangle\left[\frac{\alpha^{2}}{3}\langle\phi\rangle^{2}+\frac{\hbar \alpha^{3}}{8 \pi a}\langle\phi\rangle+\frac{\hbar \alpha^{2}}{48 a^{2}}\right]=0 .
$$

There will be non-trivial solution of Eq.(22) if the sum of the terms between brackets vanishes. However there is not $\bar{a}$ (with respect to the orders of $\alpha$ ) which satisfies Eq.(22). Therefore the minimum of $V_{e f}$ is satisfied for

$$
\langle\phi\rangle=0 .
$$

Eq.(23) shows that the vacuum is non-degenerate, and therefore spontaneous symmetry breaking cannot occur. Actually, the boundary conditions allow just one constant vacuum solution, $\langle\phi\rangle=0$. If the result had been $\langle\phi\rangle=$ constant $\neq 0$, the effective potential would not have been used and a solution $\langle\phi\rangle=\phi_{0}(x)$ would have been expected because the boundary conditions break the translational invariance.

Although Eq.(20) is finite, that is not a final result because the coupling constant in it is an arbitrary parameter. Therefore we must fit it to the renormalized coupling constant using the renormalization condition Eq.(8) to get $\lambda=\lambda_{R}$. by

The renormalized mass of the scalar field is given

$$
\left.\frac{d^{2} V_{e f}}{d \phi_{c l}^{2}}\right|_{\phi_{c}=\langle\phi\rangle=0}=\frac{\hbar \lambda_{R}}{96 a^{2}}=m_{R}^{2},
$$

that unlike what we expected it can be non-zero.

Eq.(24) shows if $\bar{a}^{2}$ is of order $\lambda$ then the mass will be of order $\lambda^{0}$. This result is in agreement with our initial assumption. If $\bar{a}^{2}$ is of lower order than $\lambda$ then the mass will lie far outside the expected range of validity of our approximation. It follows that there is a typical length scale given by the parameter $a$ of the theory. Within this length scale, the massless scalar field theory with self-interaction becomes massive due to boundary conditions effects.

Although topological mass has already been obtained [17 - 19] to order $\lambda$ for a theory which is massless at the tree-graph level, we stress the fact that if $a$ is small enough the theory will become one massive in order $\left(\hbar^{0}\right)$. This is because $\lambda$ and $a$ are independent parameters, so the mass term found can be of order zero-loop, even though it is a one-loop result (it is from radiative corrections). Our result of Eq.(24) is in agreement with that obtained by David J. Toms [19].

\section{Conclusion}

We have studied the theory of a massless, quartically self-interacting real scalar field satisfying homogeneous Dirichlet's boundary conditions on two infinite parallel plane surfaces separated by some small distance $a$. As a result, we infer:

i) For small $a\left(\bar{a} \propto \lambda^{n}, n>0\right)$ there is not any order of $\bar{a}$ with regard to order of $\lambda$ which leaves the ground state (vacuum) degenerate, i. e., spontaneous symmetry breaking does not occur. So, the effective potential can be used to evaluate the renormalized mass and the Casimir energy.

ii) There is a typical length scale of the finite region where massless scalar field acquires mass, i. e., if $\bar{a} \propto \lambda^{n}$, with $n \geq 1$, the theory becomes a theory of a massive real scalar field. Therefore, when $a$ becomes small enough the theory undergoes a transition from one massless to one massive. That typical length of finite region determines the mass scale of the theory. iii) Since spontaneous symmetry breaking does not occur, the mass generation is only due to the boundary condition.

Finally, we speculate that boundary conditions may be a mechanism of mass generation, i. e., that massless theories defined in finite regions of space-time become massive theories when the length of finite region is small enough. Therefore we conjecture that confinement may be a candidate for an alternative mechanism for the mass generation of quarks.

It is clear we do not claim that these are actual boundary conditions which produce the masses of the particles. We only consider boundary condition may be an alternative mechanism for particle mass generation.

\section{Acknowledgments}

J. A. Nogueira is especially grateful to Professor Olivier Piguet who has helped to clarify many obscure points. We would like to express our thanks to Dr. Manoelito Martins de Souza and to Dr. Francisco de Assis Ribas Bosco. It is a pleasure to thank Dr. Luiz C. Albuquerque for helpful comments. This work was supported in part by the National Agency for Research (CAPES)(Brazil).

\section{References}

[1] G. Kane, Modern Elementary Particles Physics (Addison-Wesley Publishing Company, Massachusetts, 1993)

[2] S. Coleman and E. Weinberg, Phys. Rev. D7(6), 1888 (1973).

[3] H. G. B. Casimir, Proc. K. Ned. Akad. Wet. 51, 793 (1948).

[4] M. J. Sparnaay, Physica 24, 751 (1958).

[5] S. K. Lamoureux, Phys. Rev. Lett. 28, 5 (1997). 
[6] U. Mohideen and A. Roy, Phys. Rev. Lett. 81, 21 (1998).

[7] E. Elizalde, Ten physical applications of spectral zeta function (Springer 1995).

[8] E. Elizalde et all, Zeta regularization techniques with applications (World Scientific 1994).

[9] A. A. Grib, S. G. Mamayev and V. M. Mostepanenko, Vacuum Quantum Effects in Strong Fields (Friedmann Laboratory Publishing, St Petersburg, 1994).

[10] V. M. Mostepanenko and N. N. Trunov, The Casimir Effect and its Applications (Clarendon Press, Oxford, 1997).

[11] G. E. Volovik, Vacuum in quantum liquids and in general relativity, gr-qc/0104046.

[12] M. Kardar and R. Golestanian, Rev. Mod. Phys. 71, 1233 (1999)

[13] M. Le Bellac, Quantum and Statistical Field Theory (Oxford SciencePublications, London, 1991).

[14] A. H. Guth, Phys. Rev. D23, 347 (1981).

[15] R. Rajaraman, Solitons and Instantons: An Introduction to Solitons and Instantons in Quantum Field Theory (North Hollamd, Holland, Amsterdam, 1982).

[16] K. Langfeld, F. Scmüser, and H. Reinhardt, Phys. Rev. D51(2), 765 (1995).

[17] L. H. Ford, Proc. R. London A368, 305 (1979).

[18] L. H. Ford and T. Yoshimura, Phys. Lett. A70, 89 (1979).

[19] D. J. Toms, Phys. Rev. D21(10), 2805 (1980).

[20] L. H. Ryder, Quantum Field Theory (Cambridge University Press, Cambridge, 1985).

[21] P. Ramond, FIELD THEORY A Modern Primer (The Benjamin/Cummings Publishing Company, Inc., Massachusetts, 1981).

[22] R. J. Rivers, Path Integral Methods in Quantum Field Theory (Cambridge University Press, Cambridge, 1987).
[23] K. Huang, Quarks Leptons $\mathcal{G}$ Gauge Fields (Word Scientific Publishing Company, Singapore, 1982).

[24] J. V. Narlikar and T. Padmanabhan, Gravity, Gauge Theories and Quantum Cosmology (D. Reidel Publishing Company, Dordrecht, 1986).

[25] J. Iliopoulos, C. Itzykson and A. Martin, Rev. Mod. Phys. 47, 165 (1975).

[26] R. Jackiw, Phys. Rev. D9(6), 1686 (1974).

[27] S. W. Hawking, Commun. Math. Phys. 55, 133 (1977).

[28] E. Myers, Phys. Rev. Lett. 59(2), 165 (1987).

[29] C. Itzykson and J. B. Zuber, Quantum Field Theory (McGraw-Hill Book Company, 4th printing, 1988), page 138 .

[30] J. R. Ruggiero, Aplicação da Regularização Analítica para Forças de Casimir (Dissertação de Mestrado IFT/UNESP, São Paulo, 1977).

[31] M. V. Cougo-Pinto, C. Farina and A. Tort, Rev. Brasileira de Ensino de Física, 22(1), 122 (2000).

[32] P. W. Milonni and Mei-li Shih, Contemporay Physics 33 (5), 323 (1992).

[33] J. A. Nogueira and A. Maia Jr., Phys. Lett. B358, 56 (1995).

[34] E. Elizalde and A. Romeo, J. Math. Phys. B30(5), 1133 (1989).

[35] R. V. Konoplich, Hadronic Journal 12, 19 (1989).

[36] J. Ambjorn and S. Wolfram, Ann. Phys. 147, 1 (1983).

[37] A. Actor, Fortschr. Phys. 35(12), 793 (1987).

[38] G. B. Arfken and H. J. Weber, Mathematical Methods for Physicists (Academic Press, San Diego, 1995), 4th Ed..

[39] W. Magnus, F. Oberhettinger, F. G. Triconi, edited by A. Erleyi, Higher Transcendetal Functions, ( $H$. Bateman Manuscript Projet, California Institute of Technology) (McGraw-Hill Book Company, New York, 1953) Vol. II, p. 9 and 23. 